\title{
Analyse dendroécologique sur des sapins (Abies alba Mill) et des épicéas (Picea abies (L) Karst) de différentes classes de défoliation dans la forêt de Vigo di Ton (Trento, Italie)
}

\author{
C Gandolfo ${ }^{1}$, L Tessier ${ }^{2 *}$ \\ 1 Istituto di Selvicoltura, Università degli Studi di Firenze, Firenze, 1-50145, Italie; \\ 2 IMEP (Institut Méditerranéen d'Écologie et de Paléocologie), UA CNRS D 1152, Faculté des \\ Sciences de Saint-Jérôme, F-13397 Marseille CEDEX 20, France
}

(Reçu le 12 juillet 1993 ; accepté le 20 octobre 1993)

\begin{abstract}
Résumé - Les variations de la croissance radiale de 2 populations d'Abies alba et d'une population de Picea abies provenant du Trentino sont étudiées en fonction de l'état actuel des houppiers. Le rythme de croissance des arbres, semblable pour les 3 populations jusqu'aux années 1960, se différencie ensuite. Après les 2 années critiques 1960 et 1970, les arbres actuellement dépérissants (classes de défoliation 2 et 3) montrent une faible vitalité tandis que les arbres sains (classes 1 et 2 ) récupèrent rapidement leur potentiel de croissance. Le calcul de modèles statistiques prenant en compte la tendance d'âge (modèles ARMA) et les relations cerne-climat (fonctions de réponse) sur 2 périodes successives permet de préciser cette évolution du rythme de croissance. La croissance théorique pour les années 1960-1987, reconstruite sur la base du modèle ARMA calculé sur les années antérieures (1927-1959) et confrontée à la croissance réelle, montre que le déficit de croissance est directement corrélé au degré de défoliation actuel des arbres. Parallèlement, les fonctions de réponse établies, pour chaque population et chaque classe de défoliation, sur 4 périodes successives décalées de 10 ans, mettent en évidence une diminution progressive de la sensibilité des arbres au climat initiée dans les années 1960 et 1970. Cette évolution des rythmes de croissance et de la sensibilité des arbres au facteur climatique semble modulée d'un arbre à l'autre par le patrimoine génétique (Abies est plus affecté que Picea) et les conditions microstationelles. L'état actuel des houppiers est le révélateur du poids de pertes de vitalité bien antérieures.
\end{abstract}

dépérissement / dendroclimatologie / Alpes / Trentino / Abies alba / Picea abies

Summary - Dendroecological analysis of fir (Abies alba Mill) and spruce (Picea abies (L) Karst) at different stages of defoliation in the forest of Vigo di Ton (Trento, Italy). Variations in radial growth of 2 Abies alba populations and one Picea abies population from the Trentino (fig1) have been studied in relation to the present state of tree crowns (table I) Calculation of statistical models (fig 2-4) taking into account age trends (ARMA modelling) and tree ring-climate relationships (response function) over 2 successive periods makes the quantification of growth rate changes possible. Similar growth trends (fig 5,6) were identified for the 3 populations until the 1960s, with differences occur-

\footnotetext{
* Correspondance et tirés à part
} 
ring thereafter. After 2 critical years, 1960 and 1970, the now dying trees (defoliation classes 2 and 3) underwent a loss of vitality, whereas the now healthy trees (defoliation classes 0 and 1) rapidly resumed their growth potential. Response functions established for each population and each defoliation class over 4 successive periods at 10 year intervals reveal a gradual decrease in tree sensitivity to climate from the years 1960 and 1970 onwards (figs 7-9). In parallel, the theoretical growth reconstructed for the period 1960-1987, on the basis of the ARMA model calculated for the previous period 1927-1959 and compared with the actual growth, shows that growth deficiency is directly correlated with the present degree of defoliation (fig 10). The present degree of defoliation appears to be the consequence of a loss of vitality that has been initiated by inimical climatic conditions. This sensitivity of trees to climate varies from one tree to another, depending on genetic inheritance (Abies is more affected than Picea) and site conditions

\section{forest decline / dendroecology / Alps / Trentino / Abies alba / Picea abies}

\section{INTRODUCTION}

Depuis quelques années la forêt de Vigo di Ton (Trento, Italie), repérée sur la carte de la figure 1, montre les symptômes les plus typiques d'un dépérissement : transparence et jaunissement des houppiers, ramification irrégulière (Schutt et al, 1983 ; Gellini et al, 1987 ; Capretti et Mugnai, 1991) et présence de parasites secondaires comme Armillaria mellea et Heterobasidion annosum (Delatour, 1989).

Le peuplement étudié, qui occupe une superficie de 178 ha, est globalement une sapinière équienne de 120-130 ans qui pousse sur un versant en exposition nordouest, entre $800 \mathrm{~m}$ et $1500 \mathrm{~m}$ d'altitude, où le sapin se mélange à l'épicéa au dessus de $1200 \mathrm{~m}$. Le sol, sur roche-mère calcaire, est assez mince, pauvre en humus. La pente est relativement forte (entre $30 \%$ et $60 \%$ ). Le climat présente des caractéristiques moyennes entre le type continental, caractérisé par une prédominance des précipitations estivales, et un type caractérisé par une distribution bimodale avec des maxima en automne et au printemps (Franzoi, 1993).

Dans cette forêt, la croissance radiale du sapin et de l'épicéa a été étudiée en fonction de la perte d'aiguilles des arbres, en tenant compte de l'influence du climat sur la largeur des cernes. La croissance annuelle en diamètre peut être considérée comme un bon indicateur de l'état de santé des arbres, mais ses variations interannuelles sont influencées par les oscillations des paramètres climatiques (Fritts, 1976 ; Schweingruber, 1988 ; Cook et Kairiukstis, 1989). La mise en évidence d'un éventuel déficit de croissance des arbres malades par rapport aux arbres sains doit donc obligatoirement prendre en compte les relations complexes entre cernes et climat.

L'objectif de l'étude est donc de séparer dans les séquences de cernes annuels la part de variation attribuable au rythme interne de la croissance et celle liée aux variations interannuelles du climat. Cette approche est menée sur des échantillons représentant les diverses classes de dépérissement.

\section{MÉTHODOLOGIE}

\section{Le matériel étudié}

L'échantillonnage a porté sur un ensemble de 21 sapins et 11 épicéas répartis en 3 populations choisies à 2 altitudes différentes : population $A A$ (Abies alba Mill de la station $A$, à $1000 \mathrm{~m}$ ), $\mathrm{AB}$ (Abies alba de la station B, à $1400 \mathrm{~m}$ ) et PB (Picea abies (L) Karst de la même station $B$ ). Le tableau I montre que chaque population n'est représentée que par une dizaine d'arbres, ce qui constitue un minimum. En contrepartie, l'échantillon est caractérisé par l'homogénéité du bio- 
tope, les 3 populations représentant 2 variantes altitudinales de la forêt de Vigo di Ton, les autres caractéristiques (pente, exposition, substrat, densité et âge du peuplement, cortège floristique) étant uniformes.

Les arbres étudiés ont été choisis parmi les dominants et les codominants et, autant que faire se peut, pris dans chacune des 4 classes de transparence du houppier normalement considérées dans l'inventaire de l'état sanitaire des forêts, conduit annuellement selon le. protocole CEE (voir numéro spécial de Allgemeine Forst Zeitschriff, 1983) sur toute la région. Malgré la normalisation proposée par ce protocole, il faut toutefois noter que cette appréciation comporte une part de subjectivité et reste imprécise.

Tableau I. Nombre d'arbres étudiés pour chaque population divisée en classes de défoliation.

Classe

$$
\begin{aligned}
& \text { Pourcentage de } \\
& \text { défoliation }
\end{aligned}
$$

\section{Station $A(1000 \mathrm{~m})$ Population AA Abies alba}

\section{Station $B(1400 \mathrm{~m})$ \\ Population $A B \quad$ Population PB \\ Abies alba \\ Picea abies}

$\begin{array}{lllll}0 & 0-10 & 2 & 0 & 3 \\ 1 & 11-25 & 5 & 4 & 3 \\ 2 & 26-60 & 3 & 2 & 5 \\ 3 & 61-99 & 1 & 4 & 0\end{array}$

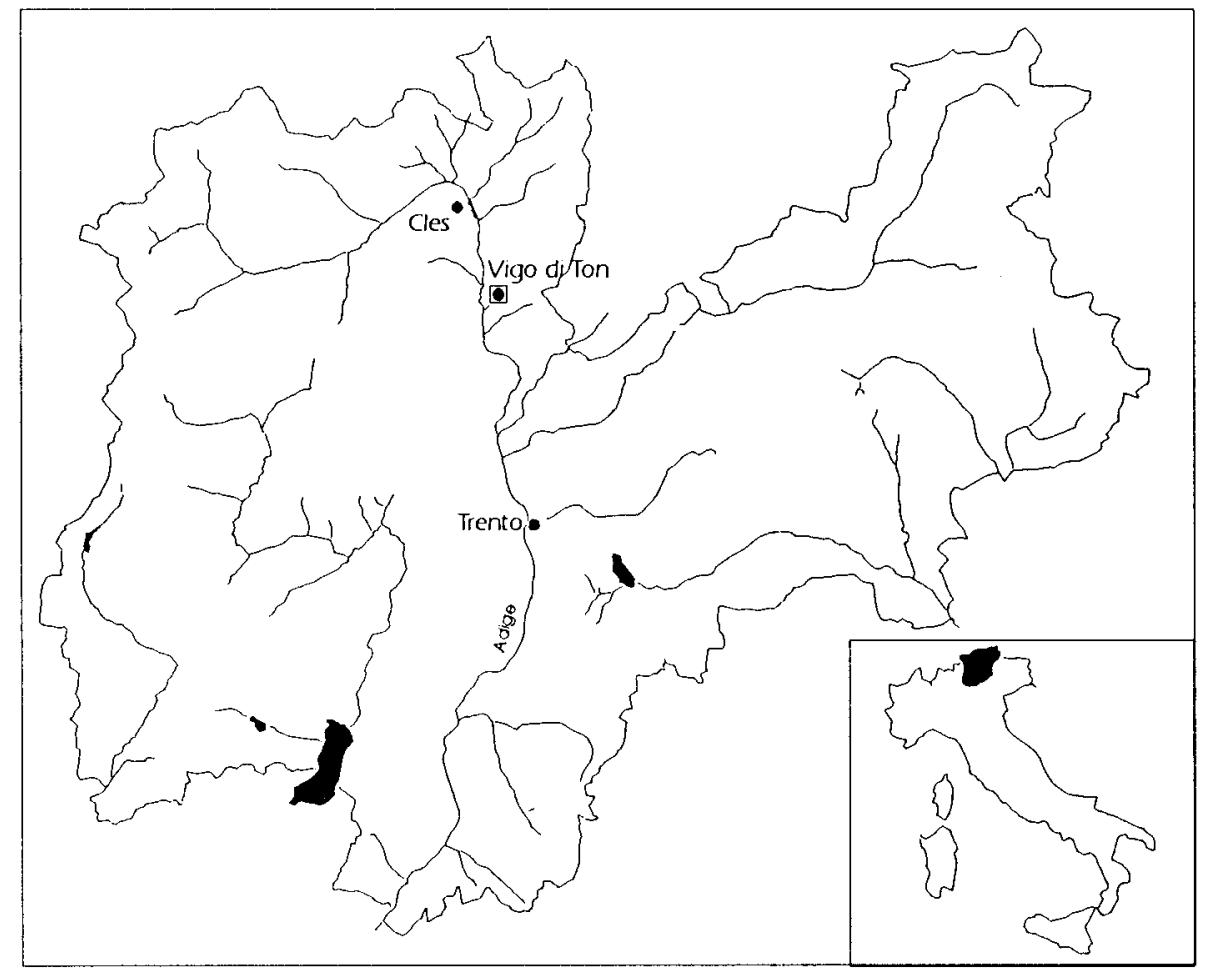

Fig 1. Localisation des postes météorologiques • et de la station d'échantillonage des arbres 
Par rapport à l'état de santé globale des forêts de la région, la forêt de Vigo di Ton se singularise par un pourcentage d'arbres dépérissants plus élevé : $35 \%$ d'arbres de classe de dépérissement 2, 3 et 4 (la classe 4 correspond aux arbres morts), par rapport aux $13 \%$ du reste de la région (Spuri Fornarini, 1991). Il faut aussi ajouter que, pour l'épicéa, on n'a pas trouvé d'arbre en classe 3 (plus de $60 \%$ de perte d'aiguilles), ce qui témoigne du meilleur état de santé de cette espèce. Parallèlement, pour le sapin de la station B, l'absence d'individus dans la classe 0 met en évidence un état sanitaire plus déficient.

De chaque arbre ont été extraites 2 carottes à $1,30 \mathrm{~m}$ de hauteur, parallèlement à la courbe de niveau. Pour les sapins de classe 3 , qui provenaient d'une coupe phytosanitaire, un disque à été prélevé à $2 \mathrm{~m}$ environ de la base. Les mesures sur les disques ont été effectuées selon les mêmes directions que celles retenues pour les carottes, en évitant les zones de croissance perturbée.

Les séries météorologiques utilisées pour l'analyse dendroclimatologique sont celles des postes de Trento $(219 \mathrm{~m})$ et Cles ( $658 \mathrm{~m})$, assez proches des sites d'étude ( $20 \mathrm{~km}$ environ), repérés dans la figure 1 et choisis pour la longueur des relevés et leur fiabilité pendant la période uti-

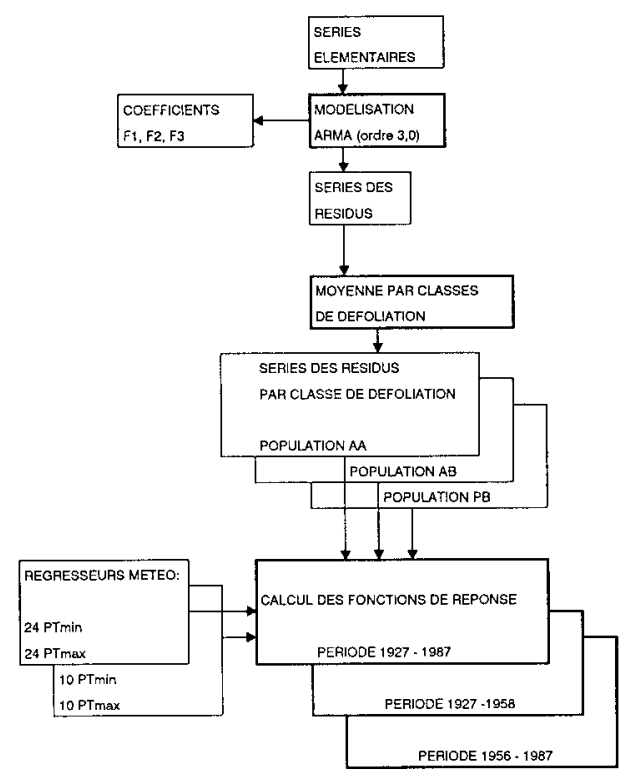

Fig 2. Organigramme du traitement des données dans le calcul des fonctions de réponse. lisée, qui couvre les années 1927-1987. Bien que situés à une altitude inférieure à celle des peuplements forestiers, ces 2 postes météorologiques représentent au mieux les variations interannuelles du climat utilisées dans l'analyse des relations cernes/climat.

\section{Traitement des données}

Les séries des largeurs de cernes relatives à la fois à 2 carottes et à 2, parfois 3 rayons de disques ont été mesurées dans un premier temps au $1 / 100 \mathrm{~mm}$. Dans un deuxième temps, les séries élémentaires ainsi obtenues ont été interdatées sur la base de tests statistiques (Aniol, 1983). Une vérification visuelle a ensuite été pratiquée sur les courbes. À ce propos, il faut observer que les arbres de classe de défoliation 3 présentaient, dans les derniers 10-12 ans, plusieurs cernes incomplets sur la circonférence, ou complètement absents, et seule la possibilité de mesurer plusieurs rayons a permis, dans la plupart des cas, l'interdatation des séries comportant ces cernes particuliers. Lorsque l'interdatation s'est avérée impossible, les séries de cernes correspondant à cette période ont été rejetées.

Pour les 3 populations, une chronologie moyenne de la largeur des cernes par classe de transparence du houppier a été construite, à partir des séries individuelles correspondant aux 2 à 3 séries élémentaires de chaque arbre. L'analyse des 10 séries moyennes obtenues a ensuite été effectuée selon 4 étapes. L'organigramme de la figure 2 décrit les 2 premières étapes.

Dans la première étape, les courbes correspondant aux chronologies des largeurs brutes ont été comparées entre elles.

Dans une deuxième étape, avant d'établir la relation cerne/climat, le signal climatique contenu dans les séries des largeurs annuelles des cernes a été isolé. Dans ce but, chaque série élémentaire des largeurs brutes a été modélisée en appliquant une procédure ARMA (Auto-Regressive Moving Average) (Box et Jenkins, 1970 ; Guiot et al, 1982 ; Guiot, 1984, 1986 ; Tessier, 1984). Toutes les séries élémentaires ont été traitées avec un modèle ARMA du même type, d'ordre 3-0. Ce type de modèle commun a été choisi après analyse des autocorrélations au sein de chaque série. La croissance de l'année test donc, pour partie, liée à la croissance des années précédentes, $t-1, t-2, t-3$; on peut donc considé- 
rer cette partie comme prédéterminée. La partie résiduelle, obtenue par soustraction de la partie modélisée à la largeur réelle mesurée, correspond à des variations aléatoires, liées à l'action du climat qui, en effet, est considéré comme variant aléatoirement d'une année à l'autre. L'expression mathématique correspondante est la suivante:

$$
\mathrm{C}_{\mathrm{t}}=\varphi_{1}{ }^{\star} \mathrm{C}_{\mathrm{t}-1}+\varphi 2^{\star} \mathrm{C}_{\mathrm{t}-2}+\varphi 3^{\star} \mathrm{C}_{\mathrm{t}-3}+\mathrm{a}_{\mathrm{t}}
$$

où $\mathrm{C}_{1}$ est la largeur du cerne au temps $t ; C_{t-1}$, $\mathrm{C}_{\mathrm{t}-2}, \mathrm{C}_{\mathrm{t}-3}$ sont les largeurs des cernes aux temps $t-1, t-2, t-3 ; \varphi_{1}, \varphi_{2}, \varphi_{3}$ sont les coefficients d'autocorrélation variant entre 0 et $1 ; a_{t}$ est le résidu aléatoire.

Une régression a ensuite été calculée entre les variables dépendantes, qui sont représentées par les 10 séries moyennes de résidus, obtenues à partir des séries de largeurs de cernes relatives aux différentes classes de perte d'aiguilles, pour les différentes populations et les variables explicatives, c'est-à-dire les 3 séries de paramètres climatiques : totaux pluviométriques mensuels $(P)$ de Cles et températures minimales et maximales mensuelles de Trento (Tmax, Tmin), considérées à partir d'octobre de l'année qui précède la construction du cerne $(n-1)$ jusqu'à septembre de l'année relative à cette construction (n). Les températures extrêmes ont été préférées aux températures moyennes dans la mesure où elles expriment plus exactement un facteur limitant potentiel.

Deux combinaisons de variables explicatives ont été utilisées. Dans un premier temps les 24 régresseurs mensuels d'octobre $n$-1 à septembre $n$; dans un deuxième, les seuls 10 régresseurs mensuels (5 précipitations et 5 températures) de la période de végétation $(n)$, de mai à septembre. Cette deuxième combinaison de variables explicatives a été choisie en fonction des résultats obtenus dans la première approche. En effet, l'utilisation de 24 variables explicatives fournit des fonctions de réponse globalement peu significatives, la séquence d'octobre $n-1$ à avril $n$ n'intervenant pratiquement pas. Le calcul fait appel à une analyse en composantes principales préalable des variables explicatives. La régression est ensuite calculée sur les composantes principales sélectionnées au seuil produit des valeurs propres (PVP) supérieur à 1 (Guiot, 1990).

Trois fonctions de réponse ont donc été établies : 1 sur la période globale1927-1987 et 2 autres sur les 2 sous-périodes d'égale longueur
1927-1958 et 1956-1987. Ces 2 périodes successives ont été choisies afin de mettre en évidence une évolution possible de la réponse des arbres au climat en fonction d'un dépérissement ayant pu évoluer progressivement dans le temps.

Les tests de signification des fonctions de réponse font appel à la méthode «boot-strap» (Diaconis et Éfron, 1983 ; Guiot, 1990). Le calcul de la fonction de réponse est répété 50 fois sur des ensembles d'années tirées au sort parmi la totalité des années de la période considérée. Chaque régression fournit un ensemble de 24 ou 10 coefficients de régression partiels. Le rapport de leur valeur moyenne $(\mathrm{rm})$ à leur écart type $(S)$ à l'issue des 50 tirages établit la signification de chaque coefficient. La signification globale est établie de la même façon sur la base du rapport du coefficient de corrélation moyen $(R m)$ entre la série des valeurs réelles et la série des largeurs estimées du climat à partir des coefficients de régression partielle obtenus à chaque tirage, à son écart type $(S)$ calculé sur les 50 tirages au sort.

Une troisième étape, dont l'organigramme est représenté dans la figure 3 , a tenté de prendre en compte l'évolution temporelle des relations cerne/climat pour les 10 chronologies moyennes. Les fonctions de réponse mettant en œuvre 24 régresseurs ont été établies sur 4 périodes de 32 ans chacune, décalées de 10 (9 les 2 dernières) : 1927-1958; 1937-1968; 1947-1978; 1956-1987. Pour chaque couple de paramètres climatiques ( $\mathrm{P}$-Tmax et P-Tmin), une matrice 24 $x 40$ a été constituée à partir des rapports $(\mathrm{rm} / \mathrm{s})$ obtenus pour chaque fonction de réponse.

Cette matrice a été soumise à une analyse en composantes principales à partir de laquelle ont été obtenus, d'une part, les plans factoriels correspondant aux 2 premières composantes qui expliquent un maximum de la variance, d'autre part une classification hiérarchique établie sur les composantes principales sélectionnées au seuil produit des valeurs propres (PVP) supérieur à 1 .

La quatrième phase prend en compte le caractère évolutif du dépérissement des arbres. Pour chaque chronologie moyenne, la croissance des années les plus récentes (1960-1987) a été reconstruite sur la base du modèle ARMA établi pour la période antérieure (1927--1959). À cette dernière période correspond un état de santé des peuplements supposé meilleur. L'objectif est ensuite de comparer les données reconstruites aux données réelles. Compte tenu du caractère 


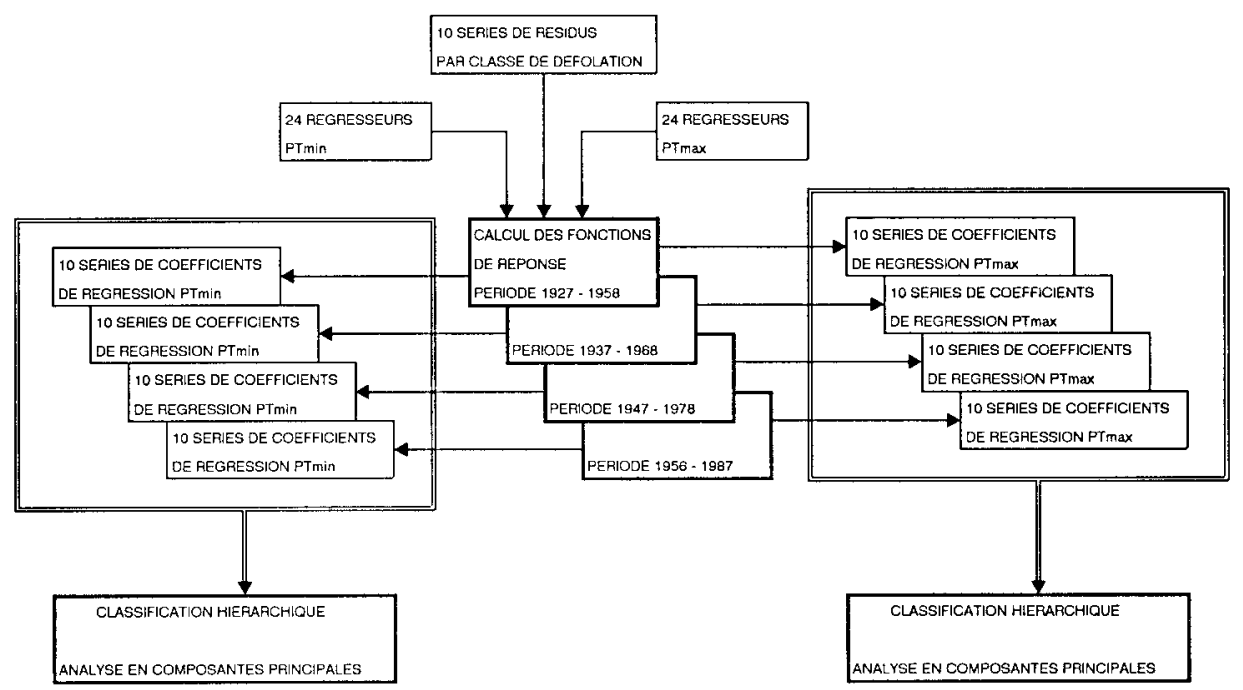

Fig 3. Organigramme du traitement des données dans l'analyse temporelle des relations cerne/climat.

équien des peuplements étudiés et de l'âge des arbres (120 ans environ), la période utilisée (19271987) écarte toute la séquence de cernes relative à la phase juvénile de la croissance des arbres. Le schéma suivi dans cette étape est indiqué dans l'organigramme de la figure 4.

Ce type précis d'analyse n'est pas nouveau dans le domaine des recherches dendroécologiques destinées à mettre en évidence un éventuel déficit de croissance lié au dépérissement des forêts et il a été déjà appliqué dans plusieurs études (Cook et al, 1987 ; Eckstein et Sass, 1989 ; Tessier et al, 1990).

\section{RESULTATS}

\section{Analyse des séries des cernes}

Le phénomène le plus évident, représenté dans la figure 5 , est la divergence, quels que soient l'essence ou le site, des courbes relatives aux différentes classes de défoliation. Ce phénomène, intervient pour le sapin dès 1960 et s'accentue après 1970 . Pour l'épicéa, la divergence n'intervient net- tement qu'à partir de 1970. Les années 1960 et 1970 sont caractérisées, pour toutes les populations, par une chute de la croissance qui affecte plus fortement les sapins de la station $A(A A)$, à $1000 \mathrm{~m}$ d'altitude. Par ailleurs, et le phénomène est surtout

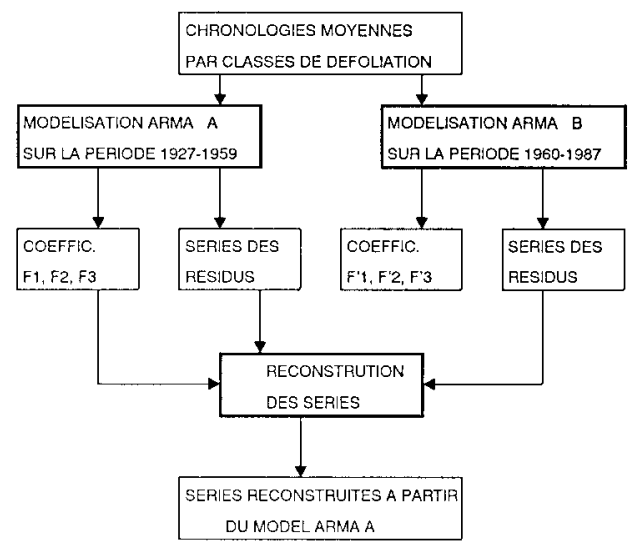

Fig 4. Organigramme du traitement des donnees dans l'estimation des séries de largeurs de cernes pour les années 1960-1987 à partir du modèle ARMA des années 1927-1959. 
Population AA

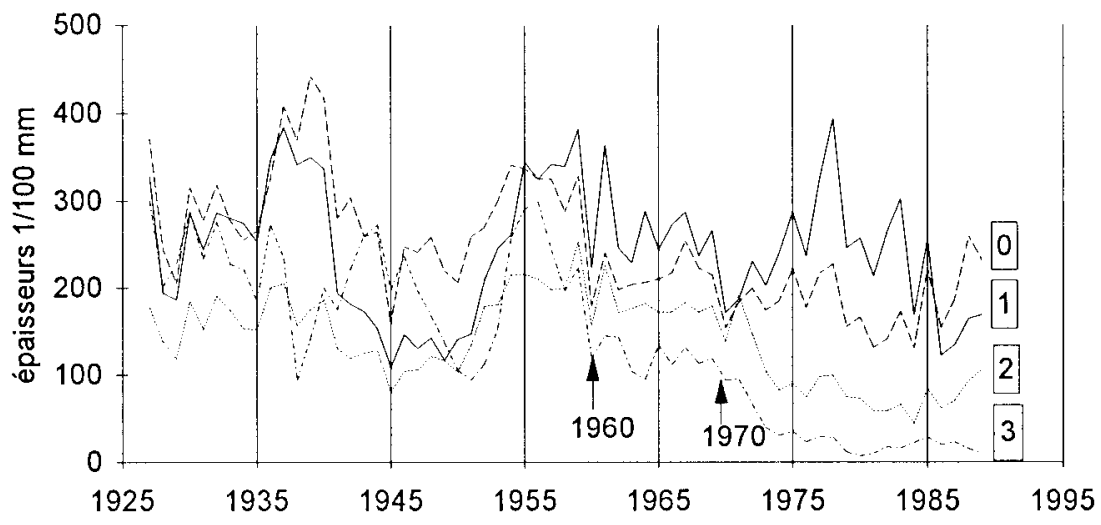

Population $A B$

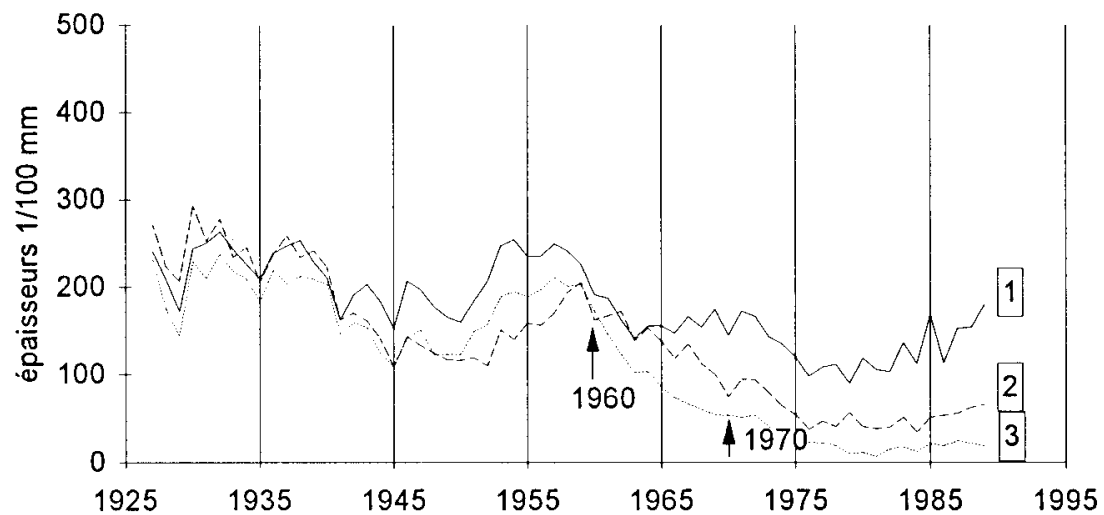

Population PB

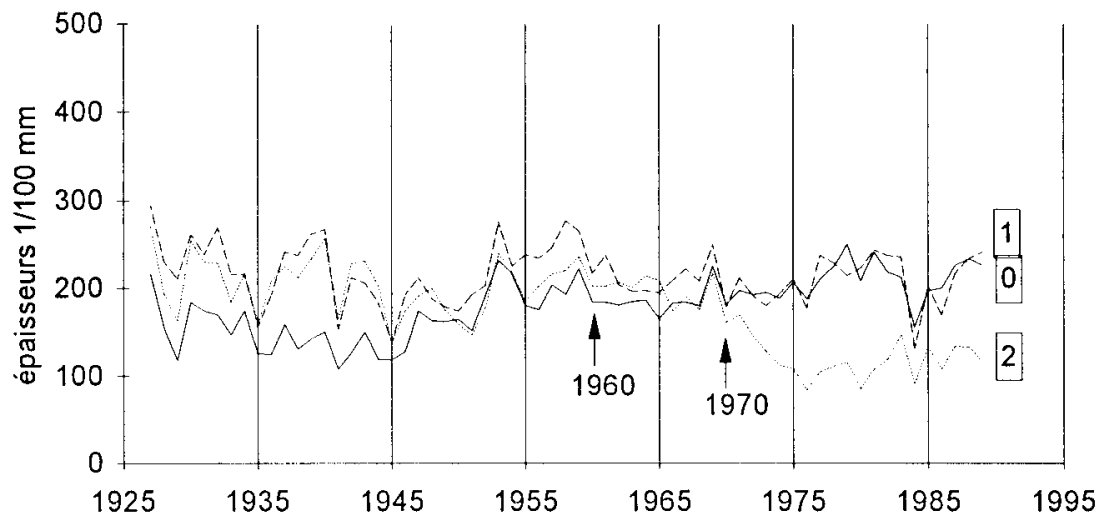

Fig 5. Chronologies moyennes par classe de défoliation. $A A=A b i e s$ alba de la station $A(1000 \mathrm{~m}$ d'altitude); $\mathrm{AB}=$ Abies alba de la station $\mathrm{B}(1400 \mathrm{~m}$ d'altitude) ; $\mathrm{PB}=$ Picea abies de la station $\mathrm{B}$ ( $1400 \mathrm{~m}$ d'altitude). Les numéros $0,1,2,3$ indiquent les classes de dépérissement. 
net pour la période postérieure à 1970 , les arbres les moins dépérissants (classes 0 et 1) récupèrent immédiatement leur potentiel de croissance alors que les arbres dépérissants (classes 2 et 3) amorcent une chute progressive de la croissance.

\section{Analyse des relations cerne/climat}

Les séries de résidus, supposées représenter au mieux l'impact des variations interannuelles du climat sur la croissance, sont caractérisées par une nette diminution de la variabilité interannuelle à partir des années 1960 et 1970, mise en évidence par la figure 6 . Cette diminution est directement liée au dépérissement puisqu'elle affecte surtout les séries correspondant aux classes de défoliation 2 et 3 .

Les résultats des différentes fonctions de réponse sont regroupés dans le tableau II. Ces résultats sont cohérents avec ceux fournis par la seule analyse des séries de résidus. Globalement, sur la totalité de la période analysée (1927-1987), la fonction de réponse n'est presque jamais significative au seuil $95 \%$. Il en est de même sur la période 1956-1987. En revanche, sur la période 1927-1958 quelques fonctions de réponse deviennent significatives au seuil de $95 \%$ et plus; elles concernent surtout l'épicéa, dont les fonctions de réponse sont représentées en figure 7 et, en particulier, la combinaison des 10 régresseurs P-Tmin.

Seules les relations avec les précipitations de mai, juillet et septembre ressortent de façon significative. En juillet et septembre, les précipitations interviennent directement quels que soient l'essence et le couple de paramètres climatiques ( $\mathrm{P}-\mathrm{T}$ max ou P-Tmin). En mai, l'intervention inverse des précipitations concerne exclusivement l'épicéa.

Les températures (seulement minimales) jouent un rôle très discret avec une intervention inverse en juillet conjointement avec les précipitations de la même période qui interviennent inversement. Cette combinaison de paramètres agit de la même façon pour les 2 essences, mais avec un niveau de signification statistique plus élevé dans le cas de l'épicéa. En mai, l'intervention couplée des précipitations (relation inverse) et des températures maximales (relation directe) concerne exclusivement l'épicéa.

\section{Analyse temporelle de la relation cerne/climat}

Les résultats rassemblés dans le tableau II mettent en évidence une évolution dans le temps de la réponse au climat des 3 peuplements. La relation non significative sur la période totale est liée à la prise en compte globale des 2 périodes successives, la première caractérisée par des relations cerne/climat significatives, la seconde, par des relations non significatives. L'analyse sur 4 périodes chevauchantes de la relation cerne/climat précise ces premières observations.

L'analyse hiérarchique, effectuée sur l'ensemble des fonctions de réponse obtenues et présentée dans la figure 8 , montre que la discrimination la plus importante est liée à la séquence temporelle, aussi bien avec le couple P-Tmax qu'avec le couple P-Tmin. Les fonctions de réponse relatives à la première période 1927-1958 s'agrègent à un même niveau, dans le même groupe hié-

Fig 6. Courbes de la variation des résidus obtenues à partir de la modélisation ARMA. Les 2 lignes horizontales représentent la valeur moyenne \pm un écart type. L'échelle des y est identique pour toutes les courbes et indique des variations par rapport à la moyenne qui est égale à 0 . Les 3 populations sont indiquées de la même façon que dans la figure 5 . 
Population AA
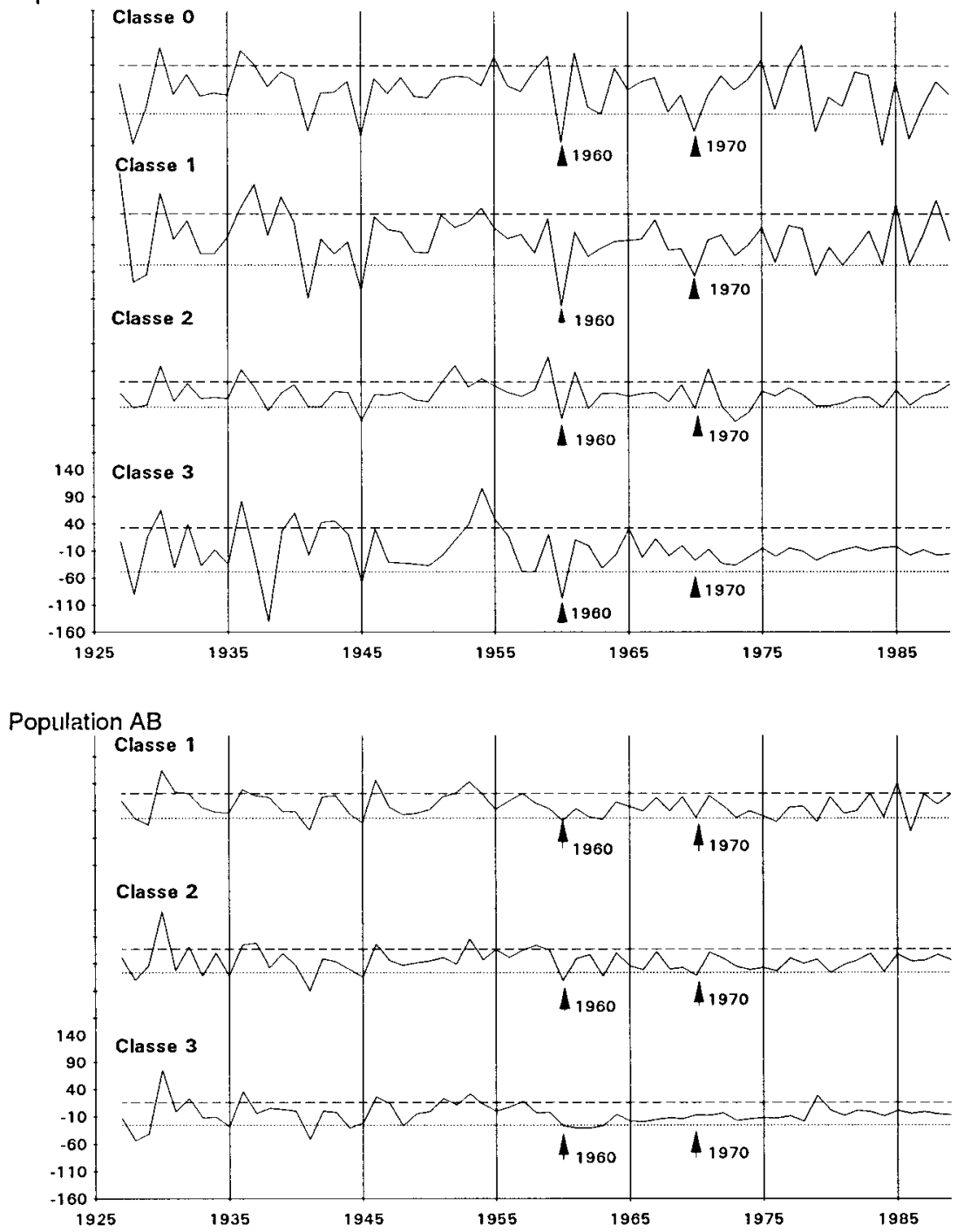

\section{Population PB}

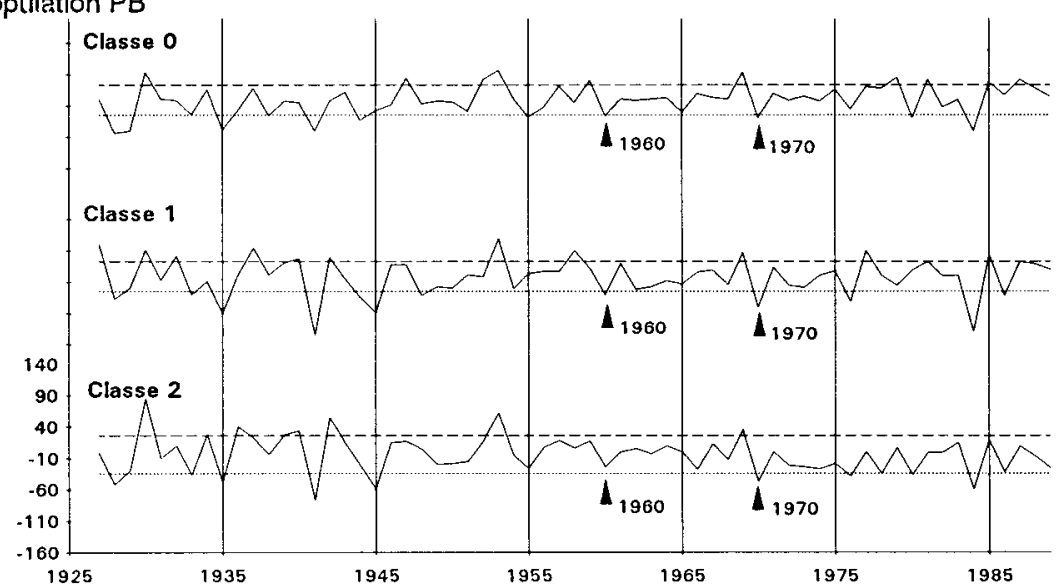




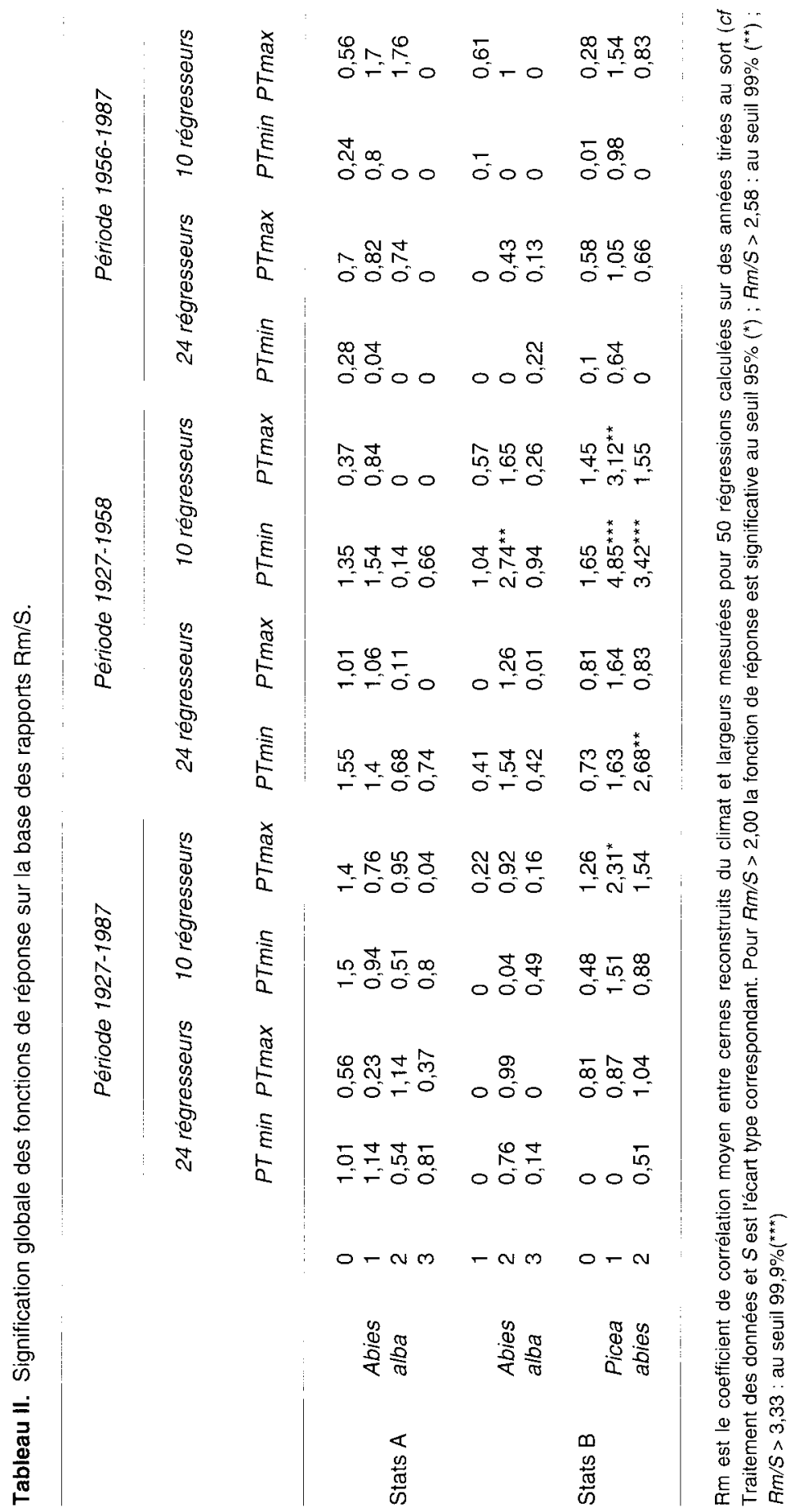


rarchique, quelles que soient la population et la classe de défoliation. Pour toutes les autres fonctions de réponse, la classification ne met pas en évidence des sépara-

\section{PTmin}

\section{CLASSE O}

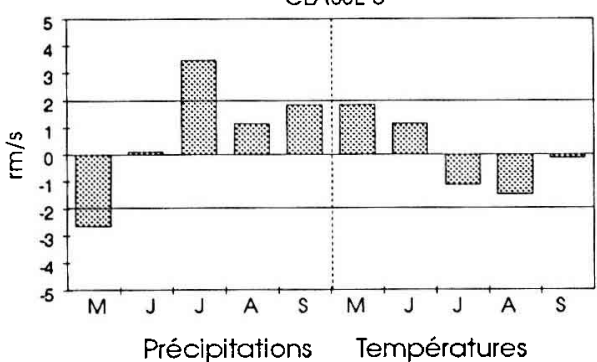

CLASSE 1

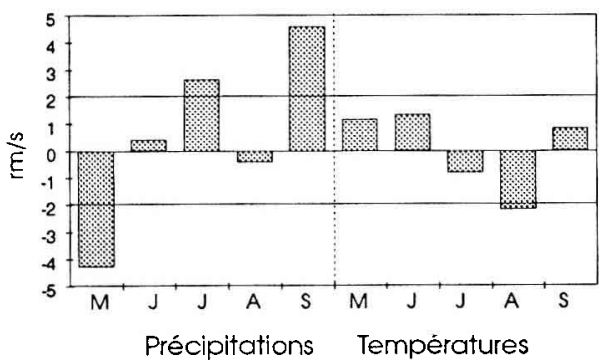

CLASSE 2

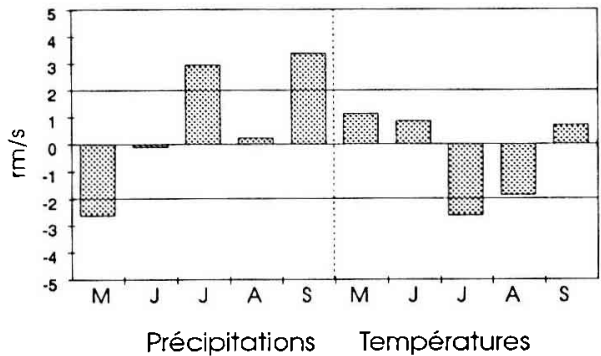

tions nettes entre groupes, mais plutôt un continuum qui, cependant, ne peut être que difficilement mis en parallèle avec les séquences temporelles successives.

\section{PTmax}
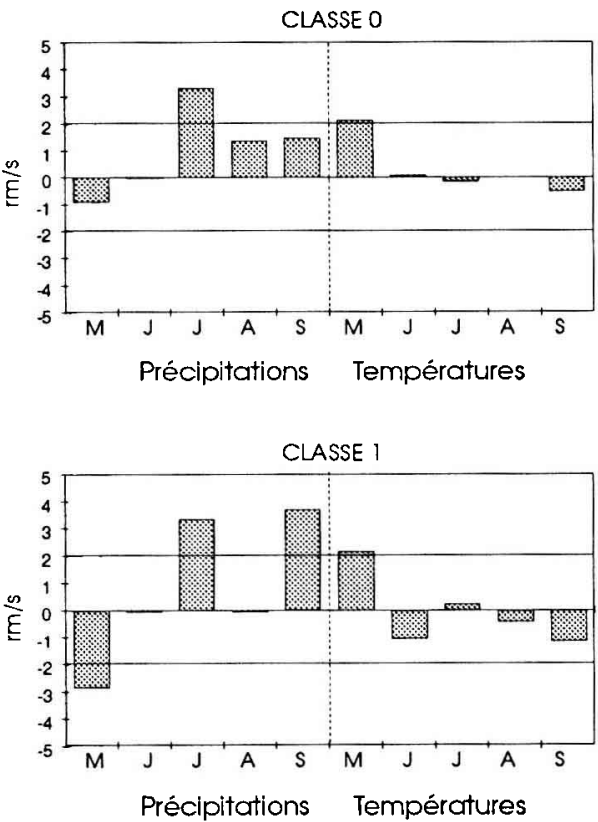

CLASSE 2

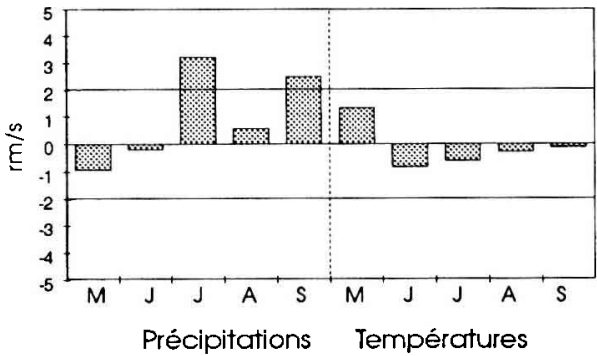

Fig 7. Profils des fonctions de réponse calculées pour la population PB (Picea abies de la station B, à $1400 \mathrm{~m}$ d'altitude) sur la période 1927-1958 avec 10 régresseurs climatiques. PTmin : régression calculée avec le couple de variables explicatives Précipitations et Températures minimales. PTmax: régression calculée avec le couple de variables explicatives Précipitations et Températures maximales. L'axe y indique la signification de la relation cerne-climat établie sur la base du rapport $R \mathrm{~m} / \mathrm{s}$ où $R m$ est le coefficient de régression moyen calculé pour chaque mois dans les 50 tirages au sort (cfle traitement des données) et s est son écart type à l'issue des 50 tirages. Sur l'axe $x$ sont représentés les mois de la période de végétation de mai à septembre. 
PTmin

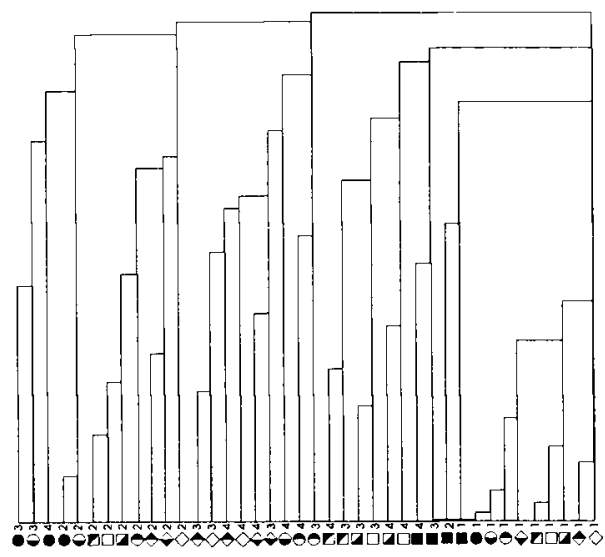

PTmax

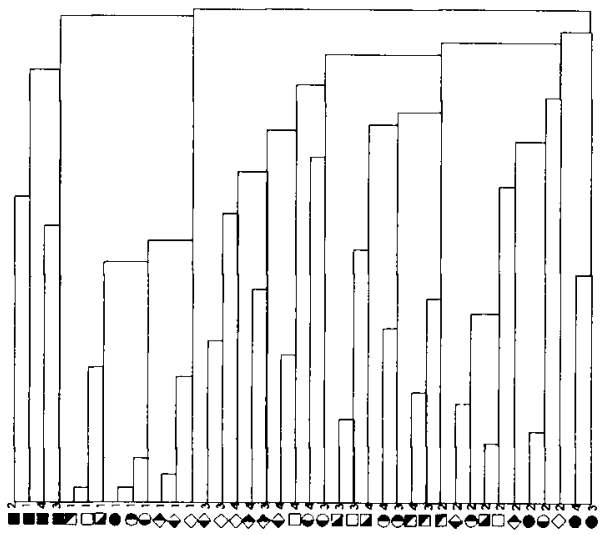

Fig 8. Analyse hiérarchique des fonctions de réponse calculées pour 4 périodes décalées de 10 ans. À chaque fonction de réponse est associé un symbole qui représente la population et la classe de dépérissement. Les chiffres 1, 2, 3, 4 indiquent les différentes périodes prises en compte. $\square \mathrm{AA}, \mathrm{OAB}, \bigcirc \mathrm{PB}, \square$ classe $0, \square$ classe $1, \square$ classe 2, classe 3, 1 1re période (19271958); $22^{e}$ période (1937-1968), $33^{e}$ période (1947-1978), $44^{e}$ période (1956-1987).
Les plans factoriels établis sur les composantes principales 1 et 2 , présentés dans la figure 9 , expliquent à eux seuls près de $50 \%$ de la variance. Le caractère discriminant de la séquence temporelle est nettement mis en évidence, aussi bien au niveau du couple P-Tmin qu'à celui du couple P. Tmax. Cependant, la distribution en fonction du temps des différents groupes de fonctions de réponse ressort plus nettement au niveau du couple P-Tmin.

Ainsi, les fonctions de réponse correspondant aux séquences temporelles successives prises isolément sont plus regroupées dans le cas du couple P-Tmin. Les fonctions de réponses regroupées de façon équivalente dans les 2 cas au niveau de la première séquence se dispersent progressivement dans le cas du couple P-Tmin alors que, dans le cas du couple P-Tmax,
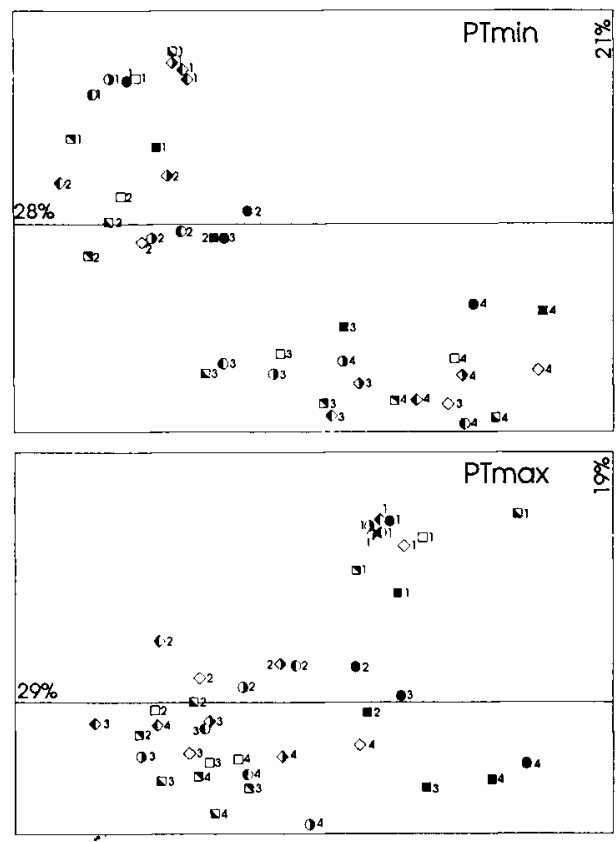

Fig 9. Analyse en composantes principales des fonctions de réponse calculées pour 4 périodes décalées de 10 ans. Les symboles sont les mêmes que ceux utilisés dans la figure 8 . 
une dispersion importante apparaît dès la seconde séquence. Par ailleurs, dans le seul cas du couple P-Tmin, les fonctions de réponse relatives aux 2 séquences initiales et celles relatives aux 2 autres séquences se séparent nettement dans les moitiés supérieure et inférieure du plan factoriel.

\section{Estimation des largeurs des cernes pour les années 1960-1987 à partir du modèle de croissance des années précédentes}

II est évident que, sur la séquence 19271959, données réelles et données reconstruites se superposent parfaitement quelles que soient la population et la classe de dépérissement, dans la mesure où les coefficients ARMA sont ceux calculés sur cette même période. Sur la période suivante (1960-1987), I'utilisation des coefficients ARMA de la période antérieure introduit des divergences entre valeurs estimées et valeurs réelles. Ces divergences affectent différemment les populations en fonction du dépérissement visuellement constaté, comme indiqué dans la figure 10.

Pour les 2 populations de sapin, la largeur estimée est presque toujours supérieure à celle des cernes réels. Ce déficit de croissance est par ailleurs plus important lorsque le degré de défoliation constaté augmente. Valeurs estimées et valeurs réelles ne sont globalement équivalentes sur la période que dans le cas de la classe de défoliation 0 .

Dans le cas de l'épicéa, le déficit de croissance n'apparaît qu'au niveau de la classe de défoliation 2 (on rappellera qu'aucun individu de la classe de défoliation 3 n'a pu être recensé).

\section{DISCUSSION}

Les analyses conduites sur les seules largeurs des cernes mettent d'abord en évi- dence que, après les années 1960 et 1970 caractérisées pour tous les arbres par une croissance réduite, seuls les arbres actuellement les plus sains (sans distinction d'espèce ni de site) ont récupéré leur potentiel de croissance. Le phénomène se manifeste d'une façon accentuée dans le cas des sapins de classe de défoliation 3 , pour lesquels la croissance des années les plus récentes est tellement compromise que, parfois, les cernes sont incomplètement formés et, quelquefois, totalement absents sur les 2 prélèvements effectués. Becker (1987) et Bert (1992) mettent en évidence un phénomène semblable pour les sapins des Vosges et du Jura.

L'analyse des courbes de croissance radiale montre également que ce phénomène de perte de vitalité a été initié quelques décennies auparavant, sans doute avant même que l'état de dépérissement ne soit apparent au niveau des houppiers.

Une chute semblable de croissance radiale a été observée entre la fin des années 1950 et le milieu des années 1960 en Suisse, pour des épicéas ayant actuellement une cime transparente (Hartmann et al, 1987). De même Becker et al (1990) trouvent que «les écarts de croissance entre populations d'arbres ont leur origine il y a plus de 50 ans et semblent surtout traduire la différenciation progressive de leur statut social».

La prolongation jusqu'en 1987 du modèle de croissance établi sur la période antérieure à 1960 précise ce phénomène. Le déficit de la croissance réelle par rapport à la croissance estimée par le modèle est directement fonction du niveau de défoliation. Le phénomène qui apparaît en 1960 s'accentue après 1970. Ces 2 années «critiques" (1960 et 1970) au point de vue de la croissance de tous les arbres introduisent un changement durable du rythme de la croissance radiale des seuls arbres répertoriés comme les plus dépérissants par l'état actuel de leur houppier. 
Population AA

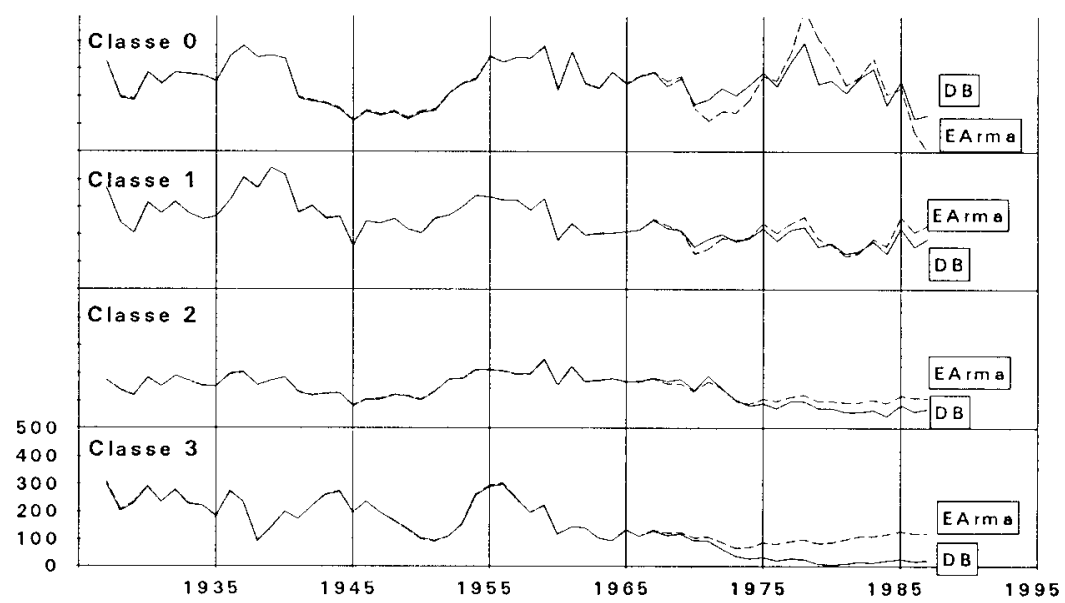

Population AB

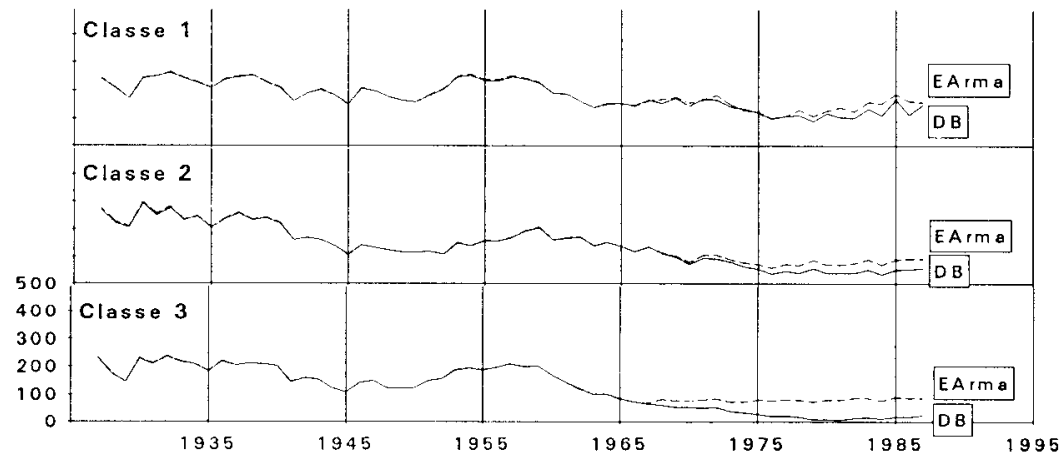

Population PB

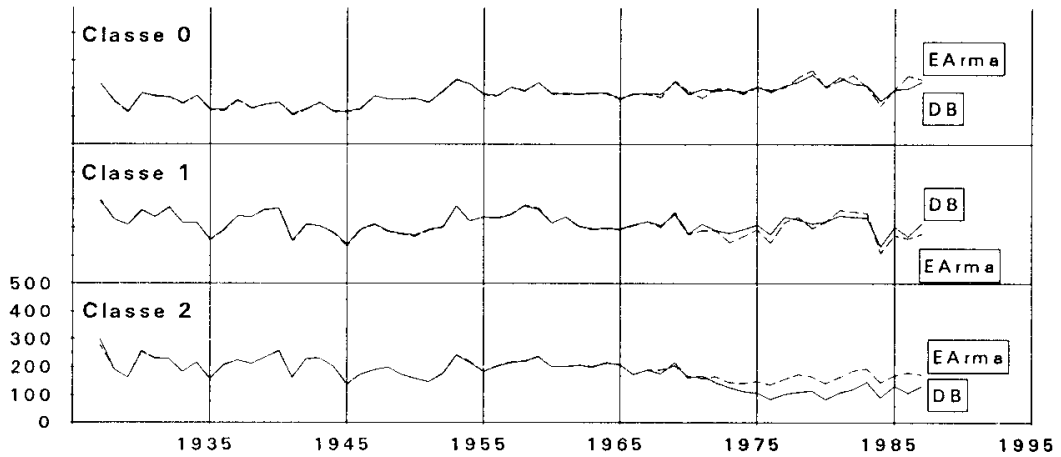

Fig 10. Courbes des données brutes (DB) et des données estimées à partir du modèle ARMA (EARMA). L'échelle utilisée est identique pour toutes les courbes. Les 3 populations sont indiquées de la même façon que dans les figures 5 et 6 . 
Parallèlement, intervient pour ces derniers arbres une baisse progressive de la sensibilité au climat. Cette évolution est mise en évidence, à la fois par la diminution, après 1960, de la variabilité interannuelle des séries des résidus issues de la modélisation ARMA, et par la diminution de la signification des fonctions de réponse relatives aux années 1956-1987 par rapport à celles de la période antérieure 19271958. Si la classification hiérarchique met surtout l'accent sur la nette séparation des fonctions de réponse relatives à la première periode par rapport aux autres prises globalement, l'analyse en composantes principales des fonctions de réponse relatives à des périodes successives décalées révèle le caractère progressif de l'évolution de la relation cerne-climat. En effet, sur le plan factoriel des 2 premières composantes principales, les fonctions de réponse relatives aux différentes périodes se regroupent assez clairement en fonction du temps.

Par ailleurs, cette même analyse en composantes principales révèle une dispersion croissante, au cours du temps, des fonctions de réponse relatives à une même période à l'intérieur de chaque groupe. $\mathrm{Ce}$ fait semble indiquer une individualisation de la réponse des arbres au climat.

Indépendamment de ces faits évolutifs, les fonctions de réponse, même si elles sont globalement assez peu significatives, mettent en évidence :

- l'influence généralisée des pluies et des températures estivales qui agissent respectivement de façon directe et de façon inverse ;

- l'exigence d'un climat sec et chaud au moment du débourrement qui est typique du seul épicéa.

Le premier aspect met l'accent sur le rôle des phénomènes d'évapotranspiration qui interviennent de la même façon sur les 2 espèces, dans les 2 sites, pendant la période de végétation. Le second met en évidence un trait particulier de l'écologie de l'épicéa qui a déjà été observé pour cette espèce en Trentino (Brugnoli et Gandolfo, 1991). Cependant, ces résultats reposant sur un échantillon restreint, sur une aire limitée, il serait hasardeux de généraliser un comportement qui caractériserait l'une ou l'autre des 2 espèces.

\section{CONCLUSIONS}

L'importance de la chute de croissance radiale des sapins et des épicéas de Vigo di Ton, qui intervient à partir de 1960 et qui s'accentue en 1970, est directement liée au degré de défoliation actuel de ces mêmes arbres. Tout se passe comme si l'état actuel du houppier n'était que l'étape ultime d'une détérioration bien antérieure des processus d'anabolisme de l'arbre. II existe donc un lien entre l'état actuel du houppier des arbres et les rythmes d'accroissement radial de ces mêmes arbres au cours des décennies antérieures. Ce phénomène affecte préférentiellement le sapin et est indépendant de la position altitudinale des arbres dans le peuplement.

Les 2 années de crise, 1960 et 1970 , qui affectent la croissance de tous les arbres n'ont pas enregistré des extrêmes climatiques tels qu'ils puissent avoir compromis la croissance radiale d'une façon significative. Si les précipitations printanières et estivales des années 1960, 1961 et 1962 ont été assez réduites, d'autres épisodes de sécheresse tout aussi importants interviennent dans les 20 dernières années. Le rôle déclencheur du facteur climatique ne peut être envisagé qu'à travers une variabilité génétique et/ou microstationelle (cf caractère superficiel du sol) qui différencierait les réactions des différents individus des peuplements.

L'analyse dans le temps de la réponse de l'arbre aux facteurs climatiques montre qu'à partir de ces 2 dates clés 1960 et 1970 la sensibilité globale des arbres aux oscil- 
lations interannuelles du climat diminue tandis que la relation cerne/climat se diversifie dans ses modalités d'un arbre à l'autre. Parallèlement le modèle de croissance établi sur la période antérieure à 1960 et appliqué aux années ultérieures fait apparaître un déficit de la croissance réelle. Ces changements ont un caractère progressif à partir des années critiques 1960 puis 1970.

\section{REFERENCES}

AAVV (1983) Neuartige Waldschäden '83. Schadstoffbelastung der Luft. Rechtsprobleme. Allg Forst Zeitschriff 51/52, 1375-1414

Aniol RW (1983) Tree ring analysis using Catras. Dendrochronologia 1, 45-53

Becker M (1987) Bilan de santé actuel et rétrospectif du sapin dans les Vosges. Étude écologique et dendrochronologique. Ann Sci For 44, 4, 379-402

Becker M, Braeker OU, Kenk G, Schneider O, Schweingruber FH (1990) Aspect des houppiers et croissance des arbres au cours des dernières décennies dans les régions frontalières d'Allemagne, de France et de Suisse. Rev For Fr 3, 284-300

Bert GD (1992) Influence du climat, des facteurs stationnels et de la pollution sur la croissance et l'état sanitaire du Sapin pectiné (Abies alba Mill) dans le Jura. Étude phytoécologique et dendrochronologique. Thèse de Doctorat. Université de Nancy I, INRA, Centre de Nancy, $200 \mathrm{p}$ et annexes

Box GFP, Jenkins GM (1970) Time Series Analysis Forecasting and Control. Hoden Day, San Francisco, $575 p$

Brugnoli A, Gandolfo C (1991) Analisi dendroclimatica sull'abete rosso (Picea abies (L) Karst) del Trentino orientale: primi risultati. Monti e Boschi 6, 51-56

Capretti P, Mugnai L (1991) Aspetti fitosanitari del bosco - osservazioni di alcune aree sperimentali in provincia di Trento. Indagine conoscitiva sullo stato sanitario delle foreste in provincia di Trento, 205-220

Cook ER, Johnson $\mathrm{H}$, Blasing TJ (1987) Forest decline: modelling the effect of climate in tree-rings. Tree Physiol 3, 27-40

Cook ER, Kairiukstis LA (1989) Methods of Dendrochronology: Applications in the Environmental Sciences. Kluwer Academic Publisher, 394 p

Delatour (1989) Impact de l'armillaire sur le système racinaire du sapin, au cours de l'évolution du peu- plement. Journée de travail DEFORPA. INRA Centre de Recherches Forestières, Nancy, vol 4, 911-929

Diaconis P. Éfron B (1983) Méthodes de calculs statistiques intensifs sur ordinateurs. Pour la Science 7 , 46-58

Eckstein D, Sass U (1989) Dendroecological assessment of decline and recovery of tir and spruce in the Bavarian Forest. IUFRO Meeting on air pollution and forest decline, 255-260

Franzoi M (1993) Danni da Cervidi al novellame di abete bianco e abete rosso nel bosco di Vigo di Ton (Trento). Università degli Studi di Firenze. Thèse non publiée, $60 \mathrm{p}$

Fritts HC (1976) Tree rings and climate. Academic Press, London, $567 \mathrm{p}$

Gellini R, Giannini R, Raddi P (1987) II deperimento del bosco: natura e cause. Atti del convegno sulle avversità del bosco e delle specie arboree da legno, 15-40

Guiot J (1984) Deux méthodes d'utilisation de l'épaisseur des cernes ligneux pour la reconstruction des paramètres climatiques anciens, exemple de leur application dans le domaine alpin. Paleogeogr Paleoclimatol Paleoecol 45, 347-368

Guiot J (1986) ARMA techniques for modelling tree-ring response to climate and for reconstructing variations of paleoclimates. Ecol Mode/ 33, 149-171

Guiot $J(1990)$ Methods and programs of statistics for paleoclimatology and paleoecology. Quantification des changements climatiques: méthodes et programmes, Monographie n. I, $253 p$

Guiot J, Tessier L., Serre-Bachet F (1982) Application de la modélisation ARMA en dendroclimatologie. $C R$ Acad Sc Paris 294, 133-136

Hartmann P, Schneider O, Petter DA, Schlaepfer R (1987) Étude des relations, chez l'épicéa, entre la largeur du cerne et l'état sanitaire de la cime. Schweiz $Z$ Forstwes 11, 923-943

Schutt $P$, Koch W, Blaschke H, Lang KJ, Schuck HJ, Summerer H (1983) So stirbt der Wald. BLV Verlagsgesellschaft, München, $95 \mathrm{p}$

Schweingruber HF (1988) Tree Rings: Basic Applications of Dendrochronology. Kluwer Academic Publischers, $276 \mathrm{p}$

Spuri Fornarini S (1991) II bosco di Vigo di Ton : vicende storiche, condizioni sanitarie e caratteristiche della vegetazione. Università degli Studi di Firenze. Thèse non publiée, $137 p$

Tessier L (1984) Dendroclimatologie et écologie de Pinus silvestris (L) et Quercus pubescens (Willd) dans le Sud-Est de la France. Thèse de Docteur ès. Sciences, Univ d'Aix-Marseille III, Fac Saint-Jerôme, $275 p$

Tessier L, Serre-Bachet F, Guiot J (1990) Pollution fluorée et croissance radiale des conifères en Maurienne (Savoie, France). Ann Sci For 47, 309-323 